\title{
Local attractors, degeneracy and analyticity: symmetry effects on the locally coupled Kuramoto model
}

\author{
Paulo F. C. Tilles, ${ }^{1,2}$ Hilda A. Cerdeira, ${ }^{1}$ and Fernando F. Ferreira ${ }^{3}$ \\ ${ }^{1}$ Instituto de Física Teórica UNESP - Universidade Estadual Paulista, \\ Rua Dr. Bento Teobaldo Ferraz, 271, Bloco II, Barra Funda, 01140-070 São Paulo, Brazil \\ ${ }^{2}$ Instituto de Física de São Carlos, Universidade de São Paulo, \\ Caixa Postal 369, 13560-970, São Carlos SP, Brazil \\ ${ }^{3}$ GRIFE, Escola de Arte, Ciências e Humanidades, Universidade de São Paulo, \\ Av. Arlindo Bettio 1000, 03828-000 São Paulo, Brazil
}

\begin{abstract}
In this work we study the local coupled Kuramoto model with periodic boundary conditions. Our main objective is to show how analytical solutions may be obtained from symmetry assumptions, and while we proceed on our endeavor we show apart from the existence of local attractors, some unexpected features resulting from the symmetry properties, such as intermittent and chaotic period phase slips, degeneracy of stable solutions and double bifurcation composition. As a result of our analysis, we show that stable fixed points in the synchronized region may be obtained with just a small amount of the existent solutions, and for a class of natural frequencies configuration we show analytical expressions for the critical synchronization coupling as a function of the number of oscillators, both exact and asymptotic.
\end{abstract}

PACS numbers: 05.45.Xt, 05.45.-a, 05.45.Jn

\section{INTRODUCTION}

Nonlinear systems tend to surprise us with their behavior contrary to what we expect from knowledge, intuition and reasoning, even when the problem appears to be rather simple, as the Kuramoto mode $\mathbf{l}^{1}$ and its local version, the locally coupled Kuramoto model (LCKM). The applicability of the model for a variety of systems that range from physics ${ }^{2.3}$, data mining 4 , neurosciences ${ }^{5.6}$, robotics $\frac{7}{6}$, animal gaits $\frac{89}{2}$, antennas $\frac{9}{9}$ and others make it important to understand the nature of unexpected results. Although the connection between the LCKM and applications is reasonably well established, the theoretical understanding is restricted to a few works $3.10-18$. Recently we studied the LCKM above the synchronization transition with periodic boundary conditions 11 . On the other hand we found a richness in the solutions which was not expected, mostly because intuition tells us that a ring of oscillators should behave as a chain for large systems. We studied how they are born and the region of phase space where the solutions exist. The complexity of the problem makes it extremely hard to analyze therefore we shall start looking at small systems with increasing difficulty to see if we can obtain some results from which we can infer large $N$ behavior, particularly when symmetry in the frequency distributions is assumed 19 .

Our objective is to calculate the behavior of the solutions for several cases where the distribution of frequencies have well determined symmetries. We will study how these solutions appear and follow them as the system size increases. To do that we shall start with the smallest system $(\mathrm{N}=3)$ which has already received the attention of Maistrenko et al ${ }^{20,21}$ and Ashwin et al ${ }^{22}$, and we are going to increase the size in the search of general properties. Obviously we shall not attempt to have an encyclopedic coverage but will show the difficulties encountered in solving the general problem with any arbitrary distribution. In doing so we have come across some results on phase slip which happens at the transition to full synchronization which seem to contradict previous results for specific symmetries ${ }^{13,14}$, which we shall discuss along the text.

The manuscript follows a route to show the loss of analyticity when one goes from small to large systems for some specific symmetry classes. To acomplish that it is organized as follows: the model is introduced in Section II; in Section III we study small systems. We start in Section III A with a well known case, that of three oscillators, where we obtain for the first time the analytic result for the dependence of the solutions on the coupling constant above synchronization; after that, in Section III B, we consider a symmetric $N=4$ case and show a general method for solving the equations for any number of oscillators, along with its difficulties. In Section IV we increase the system size and impose specular symmetry, with two natural frequencies. Under these constraints we discuss some properties for this class using a system of $N=6$ oscillators, obtaining analytical results. The analyticity limits of the model are explored in section $\mathrm{V}$ when the frequencies are obtained from a random distribution while keeping the specular symmetry. In this case we present analytical solutions only for $K_{s}$. In section VI we show some examples on how organization on the natural frequencies may lead to analytical (asymptotic) expressions for the critical synchronization coupling. A summary of the results and some possible further extensions are left for the final section.

Before ending the introduction we define some of the notation used throughout the text. When treating the bifurcations of the model we are not interested in classifying them, but on the characterization of the fixed point's stability. With that in mind, although it may seem as if 
we try to redefine well known bifurcations we name them according to the type of fixed points created at them to facilitate identification; for the fixed point classification, we call a saddle every solution containing both positive and negative eigenvalues of the Jacobian matrix and stable (unstable) node when every eigenvalue is negative (positive), regardless of their imaginary parts.

\section{PERIODIC BOUNDARY CONDITIONS: EFFECTS OF SYMMETRY}

The LCKM under periodic boundary conditions presents a rich landscape of solutions as discussed in reference 11 . From now on we shall follow the nomenclature used in that reference. The system is described by the set of equations:

$$
\dot{\theta}_{n}=\omega_{n}+K\left[\sin \left(\theta_{n-1}-\theta_{n}\right)+\sin \left(\theta_{n+1}-\theta_{n}\right)\right],
$$

for $n=1, \ldots, N$, where $\omega_{n} \in\{\omega\}_{N}$ is the set of natural frequencies. The topology of the ring is defined by the conditions $\theta_{N+1}=\theta_{1}$ and $\theta_{0}=\theta_{N}$. Alternatively, the system (11) may also be written in terms of phase differences $\phi_{n}=\theta_{n}-\theta_{n+1}$,

$$
\dot{\phi}_{n}=\omega_{n}-\omega_{n+1}+K\left[\sin \phi_{n-1}-2 \sin \phi_{n}+\sin \phi_{n+1}\right],
$$

subject to the natural identity $\sum_{n=1}^{N} \phi_{n}=0$, with the periodic boundary conditions satisfied by $\phi_{0}=\phi_{N}$ and $\phi_{N+1}=\phi_{1}$. Similarly to the case of the chain ${ }^{23}$ there is a minimum value of the coupling constant $K$ for which the system synchronizes to a common frequency $\Omega=\frac{1}{N} \sum_{j=1}^{N} \omega_{j}$. Under full synchronization the set of equations (11) can be written as

$$
\frac{\Omega-\omega_{n}}{K}=\sin \phi_{n-1}-\sin \phi_{n}, \quad n=1, \ldots, N,
$$

where $\phi_{n}=\theta_{n}-\theta_{n+1}$. The condition for the phase differences $\phi_{n}(K,\{\omega\})$ to lock depends on the number of oscillators $N$ and the value of the coupling constant in the region $K \geq K_{s}\left(K_{s}\right.$ is a unique fixed point that represents the onset of synchronization). In the synchronized state every variable $\phi_{n}$ can be written in terms of one $\phi_{n^{*}}$ arbitrarily chosen:

$$
\sin \phi_{n}=\sin \phi_{n^{*}}+\frac{1}{K} \sum_{j=n+1}^{n^{*}}\left(\Omega-\omega_{j}\right),
$$

for $n=1, \ldots, n^{*}-1$ and

$$
\sin \phi_{n}=\sin \phi_{n^{*}}-\frac{1}{K} \sum_{j=n^{*}+1}^{n}\left(\Omega-\omega_{j}\right),
$$

for $n=n^{*}+1, \ldots, N-1$, as long as we keep the right hand side on both equations in the interval $[-1,1]$. Since the identity $\sum_{j=1}^{N} \phi_{j}=0$ allows us to write $\phi_{N}$ as a sum of all the other phases $\phi_{n}$, with $n=1, \ldots, N-1$, the set of equations (3) is reduced to a single equation on two variables $\left(\phi_{n^{*}}, K\right)$ :

$$
\sin \left(\phi_{n^{*}}+\sum_{n \neq n^{*}}^{N-1} \phi_{n}\right)+\sin \phi_{n^{*}}=\frac{\sum_{j=1}^{n^{*}}\left(\omega_{j}-\Omega\right)}{K},
$$

where $\phi_{n}$ are determined by equations $4 \mathrm{a}$ and $4 \mathrm{~b}$.

Ochab and Gora ${ }^{18}$ found an equation similar to equation (5) for the topology of a ring. They introduced a parameter $p=-\sin \left(\phi_{N-1}\right)$ and vary it in the interval $[-1,1]$ to find numerical solutions for different winding numbers. They found only one branch of solutions for each winding number per $K$. In this work we treat only winding number $m=0$, which we showed in reference 11 that it has multiple solutions above synchronization. The description of the solutions equation (5) as a function of $K$ is not a simple extension of that of the chain since for the ring topology the equations for the phase differences are not independent. It is necessary to analyze simple particular cases where the small size, or particular symmetries, will give us a hint on how to proceed to the general case of randomly selected natural frequencies. This is what we shall do in the following sections.

\section{SMALL SYSTEMS ANALYSIS}

In this section we make an analysis of small systems, particularly the $N=3$ and $N=4$. We show two approaches for obtaining analytical solutions, and discuss some of the problems within this description when extended to higher number of oscillators. Unless specified on the text, from now on the values assumed by $\arcsin (x)$ are to be considered only on the interval $[-\pi / 2, \pi / 2]$.

\section{A. First non trivial case: $\mathbf{N}=\mathbf{3}$}

The first non trivial case is a system of three oscillators and natural frequencies $\omega_{1}=-\omega_{3}=\omega$ and $\omega_{2}=0$. Clearly $\Omega=0$ and the synchronized region is described by:

$$
\begin{aligned}
\sin \left(\phi_{1}+\phi_{2}\right)+\sin \phi_{1} & =\frac{\omega}{K} \\
\sin \phi_{2}-\sin \phi_{1} & =0 .
\end{aligned}
$$

Since (6b) has only two solutions,

$$
\phi_{2}^{I}=\phi_{1}, \quad \phi_{2}^{I I}=\pi-\phi_{1},
$$

it is possible to use only one phase difference $\phi_{1}=\phi$ and rewrite (6) as a single equation $K(\phi)$ for each solution:

$$
K^{I}(\phi)=\frac{\omega}{\sin \phi+\sin 2 \phi}, \quad K^{I I}(\phi)=\frac{\omega}{\sin \phi} .
$$

The function $K^{I I}(\phi)$ has the same structure as a chain of oscillators, with just a single minimum at $K^{I I}=\omega$ 


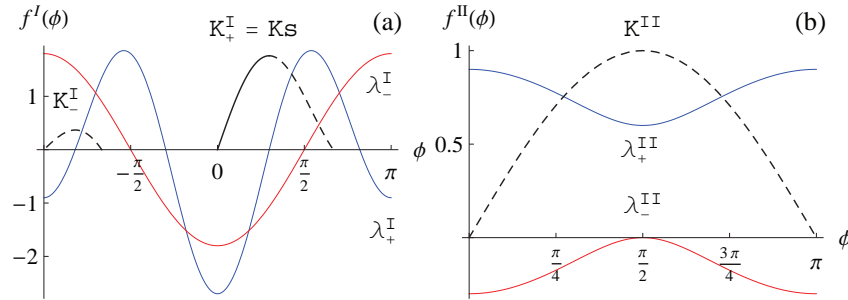

FIG. 1: (Color online) (a) Plot of the functions $f^{I}=1 / K_{ \pm}^{I}(\phi)$ where the stable solutions are shown in solid black lines and the unstable solutions in dashed lines and $\lambda_{ \pm}^{I}(\phi)$ (thin blue and red lines) for $\omega=1$ and the symmetric $N=3$ system defined by equations (6). Saddle-(stable)node bifurcation at $K_{+}^{I}=K_{s}$, the solutions are stable on the left of the bifurcation and one of the solutions is unstable, on the right. At the minimum of $K_{-}^{I}$ there is a saddle-(unstable)node bifurcation. (b) For $f^{I I}=1 / K^{I I}(\phi)$ and $\lambda_{ \pm}^{I I}(\phi)$ we see a (saddle-saddle bifurcation at the minimum of $\left.K^{I I}(\phi)\right)$.

with $\sin \phi=1$. On the other hand $K^{I}(\phi)$ has two minima for $K \geq 0$. If we define $z=\sin \phi$ these minima can be written as $K_{ \pm}^{I}=f\left(z_{ \pm}^{*}\right)$, i.e.:

$$
K_{ \pm}^{I}=\frac{\omega}{z_{ \pm}^{*}\left(1 \pm 2 \sqrt{1-z_{ \pm}^{* 2}}\right)}
$$

where

$$
z_{+}^{*}=\sqrt{\frac{15+\sqrt{33}}{32}}, \quad z_{-}^{*}=-\sqrt{\frac{15-\sqrt{33}}{32}} .
$$

Each of the three minima gives birth to a pair of solutions for $K \geq K_{s}$, and their stability is characterized by the eigenvalues of the Jacobian matrix. When we write the equations of motion as a function of the phase differences

$$
\begin{aligned}
& \dot{\phi}_{1}=\omega-K\left[2 \sin \phi_{1}-\sin \phi_{2}+\sin \left(\phi_{1}+\phi_{2}\right)\right], \\
& \dot{\phi}_{2}=\omega+K\left[\sin \phi_{1}-2 \sin \phi_{2}-\sin \left(\phi_{1}+\phi_{2}\right)\right],
\end{aligned}
$$

the eigenvalues for each type of solution are defined by

$$
\begin{aligned}
& \lambda_{ \pm}^{I}(\phi)=K[-2 \cos \phi-\cos 2 \phi \pm(\cos \phi-\cos 2 \phi)] \\
& \lambda_{ \pm}^{I I}(\phi)=K\left(1 \pm \sqrt{1+3 \cos ^{2} \phi}\right)
\end{aligned}
$$

To analyze the stability of the system, we plot the functions $f^{I}=1 / K_{ \pm}^{I}(\phi)$ and $f^{I I}=1 / K^{I I}(\phi)$, with the corresponding eigenvalues of the Jacobian matrix versus $\phi$ in figure1. We notice that the first maximum at $K_{+}^{I}$ represents a saddle-(stable)node bifurcation: two solutions are born, one stable with $\phi \rightarrow 0$ and one unstable with $\phi \rightarrow 2 \pi / 3$ in the limit $K \rightarrow \infty$. The solutions generated at the minima $K_{-}^{I}$ (figure 10) and $K^{I I}$ (figure 10) are unstable, since the real part of $\lambda_{-}^{I}$ and $\lambda_{+}^{I I}$ is always positive: $K_{-}^{I}$ is a saddle-(unstable)node bifurcation and $K^{I I}$ is a saddle-saddle bifurcation.
For this system with just a few oscillators it is not necessary to impose symmetry properties on the natural frequencies in order to obtain an analytical description of the synchronized region. If we consider the natural frequencies to be randomly chosen, it is always possible to assign positive values to $\omega_{1}$ and $\omega_{2}$ and set $\omega_{3}=-\omega_{1}-\omega_{2}$ (for $\Omega=0$ ). With this configuration the fixed point solutions are described by the equations:

$$
\begin{aligned}
\sin \left(\phi_{1}+\phi_{2}\right)+\sin \phi_{2} & =\frac{\omega_{1}+\omega_{2}}{K}, \\
\sin \phi_{1}-\sin \phi_{2} & =-\frac{\omega_{2}}{K} .
\end{aligned}
$$

A suitable manipulation of equations (14) enable us to write a single equation for $\phi_{2}$,

$$
\begin{aligned}
& \sin ^{2} \phi_{2}\left[1-\left(\sin \phi_{2}-\omega_{2} x\right)^{2}\right]= \\
& \quad\left[\left(\omega_{1}+\omega_{2}\right) x-\sin \phi_{2}-\left(\sin \phi_{2}-\omega_{2} x\right) \cos \phi_{2}\right]^{2},
\end{aligned}
$$

where $x=K^{-1}$. Since (15) is a polynomial of second order in $x$, we find solutions in the same fashion as equation (8), i.e., functional forms $K^{-1}\left(\phi_{2}\right)$ describing all the fixed points on the synchronized region:

$$
\begin{aligned}
& K_{ \pm}^{-1}\left(\phi_{2}\right)= \\
& \frac{\left(\omega_{1}+2 \omega_{2}\right) \sin \phi_{2}\left(1+\cos \phi_{2}\right) \pm \sqrt{k\left(\phi_{2}\right)}}{\omega_{1}^{2}+2 \omega_{2}\left(\omega_{1}+\omega_{2}\right)\left(1+\cos \phi_{2}\right)} \\
& k\left(\phi_{2}\right)=\sin ^{2} \phi_{2}\left[\omega_{1}^{2} \cos ^{2} \phi_{2}\right. \\
& \left.+2 \omega_{2}\left(\omega_{1}+\omega_{2}\right)\left(1+\cos \phi_{2}\right)\right] .
\end{aligned}
$$

For the symmetric case the fixed points are those shown in figure 1

It is now possible to see that the $K^{I I}$ solutions are born from the rightmost branch of $K^{I}$ (figure 2a), which lead to the pitchfork bifurcation description by Maistrenko et $a l . \stackrel{20}{ }$. A general configuration of natural frequencies generates the structure of fixed points shown in figure 2b, where the maxima of $K_{ \pm}^{-1}\left(\phi_{2}\right)$ appear separate, thus characterizing the presence of three bifurcations on the phase space. Unfortunately, the analytic expression for $K_{s}\left(\omega_{1}, \omega_{2}\right)$ does not admit a simple representation and to best of our knowledge it has not yet been reported.

\section{B. $\mathrm{N}=4$ with specular symmetry}

Based on the same assumptions as the previous case, we shall start analyzing the ring with $N=4$ oscillators where the configuration of frequencies follow a prescribed symmetry. It is worth mentioning that Maistrenko et al $\stackrel{21}{n}$ have already studied the case of $\mathrm{N}=4$ for the full connected Kuramoto model. We treat here the case when the oscillators present specular symmetry on the natural frequencies, with $\omega_{3}=-\omega_{2}, \omega_{4}=-\omega_{1}$, and both $\omega_{1}$ and $\omega_{2}$ positives.

The symmetry in the equations imposes $\sin \phi_{1}=$ $\sin \phi_{3}$, with solutions

$$
\phi_{3}^{I}=\phi_{1}, \quad \phi_{3}^{I I}=\pi-\phi_{1} .
$$



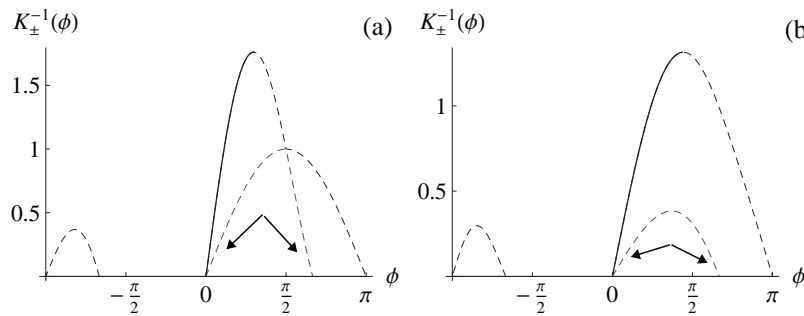

FIG. 2: (Color online) General solution $K_{+}^{-1}\left(\phi_{2}\right)$ representing the fixed points of the $N=3$ system. Stable (unstable) branches are represented by solid (dashed) lines. (a) Complete solution for the symmetric case shown in figure 1 where it is possible to see a pitchfork bifurcation at $\phi_{2}=\pi / 2$. (b) General structure of the synchronized region for a configuration of natural frequencies without symmetries: $\omega_{1}=1$ and $\omega_{2}=1 / 2$. The six fixed points solutions come from three different bifurcations. The branches that correspond to $K_{-}^{-1}$ are indicated by arrows. The others branches correspond to $K_{+}^{-1}$

For the $\phi_{3}^{I I}$ case, the equations in the synchronized state,

$$
\sin \left(\pi+\phi_{2}\right)+\sin \phi_{1}=\frac{\omega_{1}}{K}, \quad \sin \phi_{2}-\sin \phi_{1}=\frac{\omega_{2}}{K},
$$

permit the existence of a solution only if $\omega_{2}=-\omega_{1}$. As this case belongs to a more general scenario described in the next section, we will consider only the $\phi_{3}^{I}$ solution, whose synchronized region is described by the equations:

$$
\begin{aligned}
\sin \left(2 \phi_{1}+\phi_{2}\right)+\sin \phi_{2} & =\frac{\omega_{1}+\omega_{2}}{K} \\
\sin \phi_{2}-\sin \phi_{1} & =\frac{\omega_{2}}{K}
\end{aligned}
$$

After some manipulation, equation (19a) can be written as

$$
\begin{aligned}
& \sin ^{2} \phi_{1}\left(1-\sin ^{2} \phi_{1}\right)\left(1-\sin ^{2} \phi_{2}\right)= \\
& \quad\left[\frac{\omega_{1}+\omega_{2}}{2 K}-\sin \phi_{2}\left(1-\sin ^{2} \phi_{1}\right)\right]^{2} .
\end{aligned}
$$

If we would like to search for a solution of the form $K^{-1}\left(\phi_{2}\right)$, as it was made in the case $N=3$, equation (20) shows that it is necessary to obtain the roots of a fourth order polynomial. However, a more accurate investigation shows that if we define $z=\sin \phi_{2}$, it is possible to write a third order polynomial,

$$
\begin{gathered}
z^{3}\left(y_{2}-y_{1}\right)-z^{2} y_{2}\left(3 y_{2}-2 y_{1}\right)+z\left(3 y_{2}^{3}-y_{1} y_{2}^{2}+y_{1}-y_{2}\right) \\
-\frac{1}{4}\left(4 y_{2}^{4}-3 y_{2}^{2}+y_{1}^{2}+2 y_{1} y_{2}\right)=0
\end{gathered}
$$

where $y_{1,2}=\omega_{1,2} / K$. On this slightly different approach, the fixed point solutions come as the roots of equation (21), namely,

$$
\begin{aligned}
& r_{1}(K)=\frac{2 \omega_{1} \omega_{2}-3 \omega_{2}^{2}}{3 K\left(\omega_{1}-\omega_{2}\right)}+\frac{4 \omega_{1}^{2} \omega_{2}^{2}+12 K^{2}\left(\omega_{1}-\omega_{2}\right)^{2}+K^{4} f_{0}^{2 / 3}(K)}{6 K^{3}\left(\omega_{1}-\omega_{2}\right) f_{0}^{1 / 3}(K)} \\
& r_{2}^{ \pm}(K)=\frac{2 \omega_{1} \omega_{2}-3 \omega_{2}^{2}}{3 K\left(\omega_{1}-\omega_{2}\right)}+\frac{-4(1 \pm i \sqrt{3})\left[3 K^{2}\left(\omega_{1}-\omega_{2}\right)^{2}+\omega_{1}^{2} \omega_{2}^{2}\right]+(-1 \pm i \sqrt{3}) K^{4} f_{0}^{2 / 3}(K)}{12 K^{3}\left(\omega_{1}-\omega_{2}\right) f_{0}^{1 / 3}(K)}
\end{aligned}
$$

where the function $f_{0}(K)$ is defined by

$$
\begin{aligned}
& f_{0}(K)=f_{1}(K)+3 \sqrt{3} \sqrt{f_{2}(K)} \\
& f_{1}(K)=-8 \frac{\omega_{1}^{3} \omega_{2}^{3}}{K^{6}}-9 \frac{\left(\omega_{1}-\omega_{2}\right)^{2}\left(3 \omega_{1}^{2}-2 \omega_{1} \omega_{2}+3 \omega_{2}^{2}\right)}{K^{4}} \\
& f_{2}(K)=16 \frac{\omega_{1}^{3} \omega_{2}^{3}\left(\omega_{1}-\omega_{2}\right)^{4}}{K^{10}}+\frac{\left(\omega_{1}-\omega_{2}\right)^{4}\left(27 \omega_{1}^{4}-36 \omega_{1}^{3} \omega_{2}+2 \omega_{1}^{2} \omega_{2}^{2}-36 \omega_{1} \omega_{2}^{3}+27 \omega_{2}^{4}\right)}{K^{8}}-64 \frac{\left(\omega_{1}-\omega_{2}\right)^{6}}{K^{6}} .
\end{aligned}
$$

The roots $r_{1}(K)$ and $r_{2}^{-}(K)$ give the locked solutions of $\sin \phi_{2}(K)$ for $K \geq K_{s}$, but as $\left|r_{2}^{+}\right|$is always greater than 1 this root is not a valid representation of $\sin \phi_{2}$. From these solutions it is possible to build a bifurcation diagram for the system once we know the stability of the solutions. Bearing in mind that we are searching for simple analytical expressions, we will consider a reduction of the phase space dynamics. With a proper set of initial conditions, i.e., $\phi_{3}(t=0)=\phi_{1}(t=0)$, with $\phi_{1}(t=0)$ and $\phi_{2}(t=0)$ randomly generated, the dynamics is set to occur in a 2-dimensional symmetric manifold. Now, if we follow the same procedure of the previous section and write the equations of motion for the phase differences 

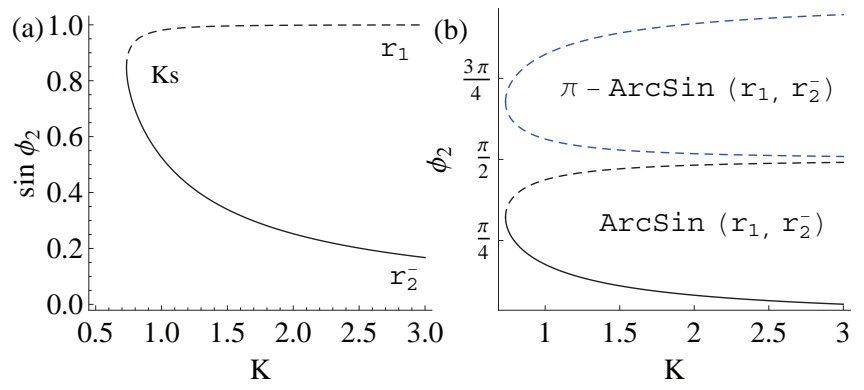

FIG. 3: (Color online) Roots $r_{1}(K)$ and $r_{2}^{-}(K)$ of (21) representing the bifurcation diagram in the synchronized region for $\omega_{1}=1$ and $\omega_{2}=1 / 3$ for the system with $\mathrm{N}=4$ defined by equations (19). Full (dashed) lines represent stable (unstable) solutions. a) Bifurcation diagram projected on the space $\sin \phi_{2} \times K$ showing the bifurcation at $K_{s}$ where two solutions are born: one stable with $\sin \phi_{2} \rightarrow 0$ and another unstable with $\sin \phi_{2} \rightarrow 1$ in the limit $K \rightarrow \infty$. b) Bifurcation diagram showing the simultaneous birth of the solutions in different regions of phase space at $K_{s}$, a saddle-(stable)node in the region $\cos \phi_{2}>0$ and an saddle-(unstable)node for $\cos \phi_{2}<0$.

$\phi_{n}$,

$$
\begin{aligned}
\dot{\phi}_{1}= & \omega_{1}-\omega_{2} \\
& -K\left[2 \sin \phi_{1}-\sin \phi_{2}+\sin \left(2 \phi_{1}+\phi_{2}\right)\right], \\
\dot{\phi}_{2}= & 2 \omega_{2}+2\left(\sin \phi_{1}-\sin \phi_{2}\right),
\end{aligned}
$$

we obtain a closed analytical expression for the eigenvalues of the Jacobian matrix,

$$
\lambda_{ \pm}=\frac{\tau \pm \sqrt{\tau^{2}-4 \Delta}}{2},
$$

where the trace $\tau$ and the determinant $\Delta$ of the matrix are given by

$$
\begin{aligned}
\tau= & -2 K \cos \phi_{1}\left[1+2 \cos \left(\phi_{1}+\phi_{2}\right)\right], \\
\Delta= & -4 K^{2} \cos ^{2}\left(\frac{\phi_{1}+\phi_{2}}{2}\right)\left[1-\cos 2 \phi_{1}\right. \\
& \left.-2 \cos \left(\phi_{1}+\phi_{2}\right)\right] .
\end{aligned}
$$

At this point we find a problem: the locked solutions are given in terms of $\sin \phi_{2}$ while the eigenvalues of the Jacobian depend on $\phi_{1}$ and $\phi_{2}$. A way out of this conflict can be reached if one notices that for each root in (22) there are two solutions for $\phi_{2}$ :

$$
\begin{aligned}
& \phi_{2}^{I}\left(r_{1}\right)=\arcsin r_{1}, \quad \phi_{2}^{I I}\left(r_{1}\right)=\pi-\arcsin r_{1}, \\
& \phi_{2}^{I}\left(r_{2}^{-}\right)=\arcsin r_{2}^{-}, \quad \phi_{2}^{I I}\left(r_{2}^{-}\right)=\pi-\arcsin r_{2}^{-} .
\end{aligned}
$$

Since $r_{1}$ and $r_{2}^{-}$do not depend on $\phi_{1}$, it is necessary to go back to equations (19) to determine the dependency of $\phi_{1}$ on $\phi_{2}$ for each solution of (27). A numerical analysis of the equations shows that $\phi_{1}=\arcsin \left(\sin \phi_{2}-\frac{\omega_{2}}{K}\right)$ leads to the solutions $\phi_{2}^{I}\left(r_{1}\right)$ and $\phi_{2}^{I}\left(r_{2}^{-}\right)$, while $\phi_{1}=$
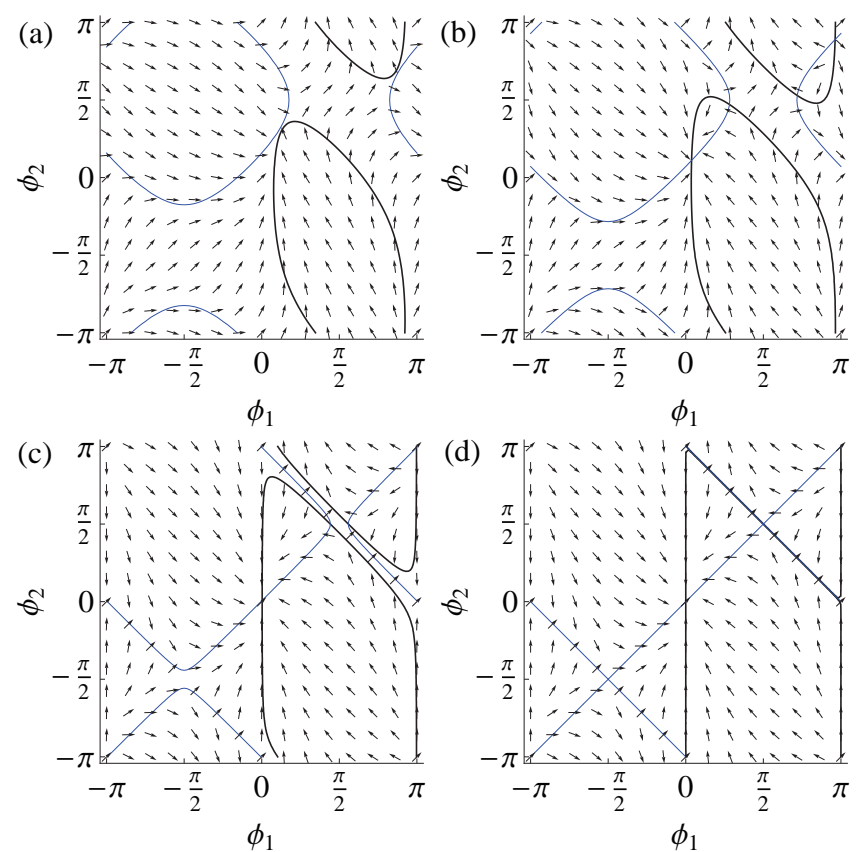

FIG. 4: (Color online) Phase space showing the evolution of the fixed points as a function of $K$ for a fixed configuration of natural frequencies $\omega_{1}=1$ and $\omega_{2}=1 / 3$ in the system with $N=4$. Black(thick) and blue(thin) lines correspond to $\dot{\phi}_{1}=0\left(\dot{\phi}_{2}=0\right)$. a) $K=0.7$ : just before $K_{s}$, we can see the simultaneous bifurcations. b) $K=1.5$ : stable node and saddle created in the region with $\cos \phi_{2}>0$ (central part of the figure), and saddle and unstable node created in the region $\cos \phi_{2}<0$, in the upper part of the figure. c) $K=20.0$ : for large values of $K$ the stable node moves towards $\left(\phi_{1}, \phi_{2}\right)=(0,0)$, the two unstable saddles go towards $(\pi / 2, \pi / 2)$ to merge into a unique saddle. d) In the limit $K \rightarrow \infty$, the unstable node moves towards $(\pi, \pi)$ while the curves deform to be a saddle at $(0, \pi)$, when $K \rightarrow \infty$.

$\pi-\arcsin \left(\sin \phi_{2}-\frac{\omega_{2}}{K}\right)$ corresponds to the complementary branches $\phi_{2}^{I I}\left(r_{1}\right)$ and $\phi_{2}^{I I}\left(r_{2}^{-}\right)$.

As a result of this analysis it is possible to observe that, while the graph $\sin \phi_{2} \times K$ shows $K_{s}$ as a bifurcation giving birth to two solutions (figure 3 ), the identity $\sin \phi=\sin (\pi-\phi)$ implies that for each branch of $\sin \phi_{2}$ there are actually two fixed points in the $\phi_{2} \times K$ space (figure 3 $\mathrm{b}$ ). The unexpected (though straightforward) conclusion is the feature that the $K_{s}$ bifurcation occurs simultaneously in two regions of the phase space, but the fixed points do not share the stability properties: in the region defined by $\cos \phi_{2}>0$ it bifurcates into one stable $\phi_{2}^{I}\left(r_{1}\right)$ and one unstable branch $\phi_{2}^{I}\left(r_{2}^{-}\right)$, meanwhile for $\cos \phi_{2}<0$ it has two unstable solutions, a saddle $\phi_{2}^{I I}\left(r_{2}^{-}\right)$and an unstable node $\phi_{2}^{I I}\left(r_{1}\right)$.

Now it becomes evident why we chose a reduced subspace for the dynamics: due to its lower dimensionality it is possible to visualize the evolution of the nullclines and the creation of the fixed points in the phase space, as shown in figure 4 Just before the bifurcation at $K_{s}$ it is possible to visualize the two simultane- 
ous fixed points being formed at $\left(\phi_{1}, \phi_{2}\right) \approx(0.4,1)$ and $\left(\phi_{1}, \phi_{2}\right) \approx(2.5,2.5)$ (figure 4 a). Figure 4 o $\left(K>K_{s}\right)$ shows the stable node at $\left(\phi_{1}, \phi_{2}\right) \approx(0,0.5)$ and the saddle at $\left(\phi_{1}, \phi_{2}\right) \approx(1,1.4)$ born in the region $\cos \phi_{2}>0$, as well as the saddle at $\left(\phi_{1}, \phi_{2}\right) \approx(2.5,1.5)$ and the unstable node at $\left(\phi_{1}, \phi_{2}\right) \approx(3,2.5)$. In the limit $K \rightarrow \infty$ the stable node shifts towards $(0,0)$, the two saddles move towards $(\pi / 2, \pi / 2)$ until they merge into a unique saddle, and the unstable node shifts in the direction of $(\pi, \pi)$ (figures 4 c and $4 \mathrm{~d}$ ).

For this specific system the reduced manifold contains all the solutions of the complete 3-dimensional phase space, and the stability of the fixed points does not suffer any alteration when one reduces the dimension of the dynamics. However this is not the general behavior for the LCKM when symmetries on the natural frequencies are taking into account, as we will show in the next section.
The value of the coupling constant at the synchronization transition can be obtained as the minimum of the function $K\left(\sin \phi_{2}\right)=K(z)$, such that $\left.\partial_{z} K(z)\right|_{z=z^{*}}=0$ implicitly calculated in (21) gives two solutions

$$
z_{ \pm}^{*}=\frac{\omega_{2}\left(2 \omega_{1}-3 \omega_{2}\right) \pm \sqrt{\omega_{1}^{2} \omega_{2}^{2}+3 K^{2}\left(\omega_{1}-\omega_{2}\right)^{2}}}{3 K\left(\omega_{1}-\omega_{2}\right)} .
$$

Once we take into account the two roots $z_{ \pm}^{*}$ in (21) we obtain four solutions:

$$
K_{s}^{ \pm, \pm}\left(\omega_{1}, \omega_{2}\right)= \pm \frac{1}{8 \sqrt{2}\left|\omega_{1}-\omega_{2}\right|} \sqrt{k_{0}^{ \pm}\left(\omega_{1}, \omega_{2}\right)},
$$

where the function $k_{0}^{ \pm}\left(\omega_{1}, \omega_{2}\right)$ is defined as

$$
\begin{aligned}
k_{0}^{ \pm}\left(\omega_{1}, \omega_{2}\right)= & 27 \omega_{1}^{4}-9 \omega_{1}^{3}\left(4 \omega_{2} \pm \sqrt{k_{1}\left(\omega_{1}, \omega_{2}\right)}\right)+\omega_{2} \omega_{1}^{2}\left(2 \omega_{2} \pm 5 \sqrt{k_{1}\left(\omega_{1}, \omega_{2}\right)}\right)-\omega_{1} \omega_{2}^{2}\left(36 \omega_{2} \mp 5 \sqrt{k_{1}\left(\omega_{1}, \omega_{2}\right)}\right) \\
& +9 \omega_{2}^{3}\left(3 \omega_{2} \mp \sqrt{k_{1}\left(\omega_{1}, \omega_{2}\right)}\right) \\
k_{1}\left(\omega_{1}, \omega_{2}\right)= & 9 \omega_{1}^{2}-14 \omega_{1} \omega_{2}+9 \omega_{2}^{2} .
\end{aligned}
$$

For the case considered ( $\omega_{1}$ and $\omega_{2}$ positive) the solutions $K_{s}^{ \pm,+}$are always complex conjugate pure imaginary numbers, and do not represent a physical solution. On the other hand the solutions $K_{s}^{ \pm,-}$are always real, but, since $K_{s}^{-,-}$is always negative the critical coupling at synchronization is given by

$$
K_{s}=K_{s}^{+,-}\left(\omega_{1}, \omega_{2}\right) \text {. }
$$

In the figures we have taken $\omega_{1}=1$ and $\omega_{2}=1 / 3$ for which $K_{s} \approx 0.734$.

With this two examples of small systems $(N=3$ and $N=4$ ) we gave a description of how analytical expressions for the location of the fixed points and the critical synchronization coupling may be obtained, which we summarize here: starting from equation (5), if we make the trigonometric expansion of the $\sin \phi_{N}$ term into sines and cosines of all the phase differences, then by squaring the two sides of the equation (with subtle rearrangements of the terms) it is possible to use equations (4) to write a polynomial equation either for $z=\sin \phi_{n^{*}}$ or $x=K^{-1}$. Ultimately the fixed points will come as the roots of the polynomial, and the critical synchronization coupling $K_{s}$ appears as the first minimum of the function $K\left(\phi_{n^{*}}\right)$, computed with respect to either $\phi_{n^{*}}$ or $\sin \phi_{n^{*}}$, as long as $\phi_{n^{*}}$ is chosen properly (for further information about this point see 11 ). The problem with this approach is that the degree of the polynomial increases with the number of oscillators, and for $N \geq 5$ the degree is typically too high to obtain analytical roots. Nevertheless if we make use of highly symmetrical configurations of natural frequencies the behavior of the system may be analytically explored to a large extent. This is the task that we shall undertake in the next section.

\section{TWO NATURAL FREQUENCIES}

The simplest system with nontrivial behavior one may consider is composed of only two natural frequencies. But as local coupled systems present a strong dependence on the local configurations, we will get rid of the inhomogeneities by assuming an organized distribution of the natural frequencies:

$$
\omega_{n}= \begin{cases}\omega, & \text { for } n \text { odd } \\ -\omega, & \text { for } n \text { even }\end{cases}
$$

In the synchronized region the symmetry reduces the equations to a set of constraints on the phase differences $\phi_{n}$,

$$
\sin \phi_{n}=\left\{\begin{array}{l}
\sin \phi_{1}, \text { for } n=3,5, \ldots, N-1, \\
\sin \phi_{2}, \text { for } n=4,6, \ldots, N-2 .
\end{array},\right.
$$

while $\phi_{1}$ and $\phi_{2}$ satisfy

$$
\begin{aligned}
\sin \left[\sum_{n=\text { odd }}^{N-1} \phi_{n}\left(\phi_{1}\right)+\sum_{n=\text { even }}^{N-2} \phi_{n}\left(\phi_{2}\right)\right]+\sin \phi_{1} & =\frac{\omega}{K} \\
\sin \phi_{1}-\sin \phi_{2} & =\frac{\omega}{K}
\end{aligned}
$$


A straightforward solution of this set corresponds to take $\phi_{n}=\phi_{1}$ for $n$ even, $\phi_{n}=\phi_{2}$ for $n$ odd and $\phi_{2}=-\phi_{1}$, such that (34) can be written as

$$
\sin \phi_{1}=\frac{\omega}{2 K} .
$$

As this set of solutions $\left(\phi_{n}\right)$ maximizes the left hand side of (34a), the critical synchronization coupling is determined as the first solution of equation (35), i.e.,

$$
K_{s}=\frac{\omega}{2} .
$$

Although this specific choice of the phases gives a fixed point solution valid for $K \geq K_{s}$, the synchronized region is full with solutions obtained from combinations of $\phi_{n}^{+}=\phi_{1,2}$ and $\phi_{n}^{-}=\pi-\phi_{1,2}$, in a way that a complete description of the system involves all possible combinations of the solutions to equation (33).

To obtain all the solutions (fixed points) with a prescribed symmetry, we will explore the system's symmetries to get the simplest description of the dynamics. Since equation (33) tells us that there are two independent phases, it is possible to consider proper initial conditions $\phi_{n}(t=0)$ that reduce the original $(N-1)$ dimensional system down to a 2 -dimensional set of equations. If we define

$$
\begin{aligned}
& \phi_{n}^{+}=\left\{\begin{array}{l}
\phi_{1}, \text { for } n=3,5, \ldots, N-1, \\
\phi_{2}, \text { for } n=4,6, \ldots, N-2 .
\end{array}\right. \\
& \phi_{n}^{-}=\left\{\begin{array}{l}
\pi-\phi_{1}, \text { for } n=3,5, \ldots, N-1, \\
\pi-\phi_{2}, \text { for } n=4,6, \ldots, N-2 .
\end{array}\right.
\end{aligned}
$$

the equations of motion are written as

$$
\begin{aligned}
& \dot{\phi}_{1}=2 \omega-K\left(2 \sin \phi_{1}-\sin \phi_{2}+\sin \Phi\right), \\
& \dot{\phi}_{2}=-2 \omega+2 K\left(\sin \phi_{1}-\sin \phi_{2}\right),
\end{aligned}
$$

where

$$
\begin{aligned}
\Phi & \equiv \Phi^{ \pm, \pm, \ldots}\left(\phi_{1}, \phi_{2}\right)=-\phi_{N} \\
& =\phi_{1}+\phi_{2}+\sum_{n=3,5, \ldots}^{N-1} \phi_{n}^{ \pm}\left(\phi_{1}\right)+\sum_{n=4,6, \ldots}^{N-2} \phi_{n}^{ \pm}\left(\phi_{2}\right),
\end{aligned}
$$

defines each of the $2^{N-3}$ solutions that compose the $\phi_{N}$ term, thus generating all the subspaces of the system. The stability of the solutions is described by the eigenvalues of the Jacobian matrix

$$
\lambda_{ \pm}=\frac{\tau \pm \sqrt{\tau^{2}-4 \Delta}}{2},
$$

where $\tau$, the trace and $\Delta$, the determinant are defined by

$$
\begin{aligned}
\tau= & -K\left[2\left(\cos \phi_{1}+\cos \phi_{2}\right)+\cos \Phi \partial_{\phi_{1}} \Phi\right] \\
\Delta= & 2 K^{2}\left[\cos \Phi\left(\cos \phi_{2} \partial_{\phi_{1}} \Phi-\cos \phi_{1} \partial_{\phi_{2}} \Phi\right)\right. \\
& \left.+\cos \phi_{1} \cos \phi_{2}\right] .
\end{aligned}
$$

(a)

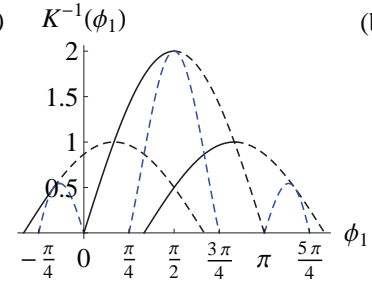

(c)

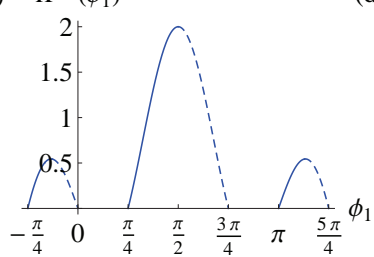

(b)

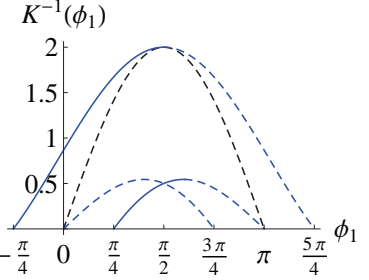

(d) $K^{-1}\left(\phi_{1}\right)$

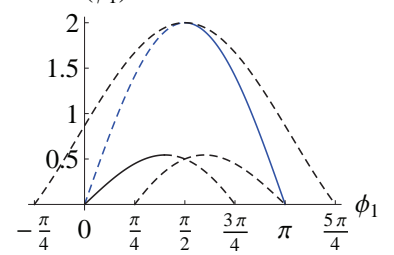

FIG. 5: (Color online) Bifurcation diagram $K^{-1}\left(\phi_{1}\right)$ showing the solutions and their stability defined in the symmetric spaces for a system with $N=6$ and $\omega=1$. Solid (dashed) lines represent stable (unstable) solutions. (a) Subspace $\Phi^{+++}$. (b) Subspace $\Phi^{-++}$. (c) Solutions $\phi_{2}^{-}$of subspace $\Phi^{+-+}$. (d) Subspace $\Phi^{-+-}$.

Starting with a $N=6$ system, the symmetrical subspaces are defined by $\Phi^{ \pm \pm \pm}$such that the equation (34a) has the following representations in each subspace:

$$
\begin{aligned}
& \Phi^{+++} \quad \rightarrow \quad \sin \left(3 \phi_{1}+2 \phi_{2}\right)+\sin \phi_{1}=\frac{\omega}{K}, \\
& \left.\begin{array}{l}
\Phi^{-++} \\
\Phi^{++-}
\end{array}\right\} \quad \rightarrow \quad-\sin \left(\phi_{1}+2 \phi_{2}\right)+\sin \phi_{1}=\frac{\omega}{K}, \\
& \Phi^{+-+} \quad \rightarrow \quad-\sin 3 \phi_{1}+\sin \phi_{1}=\frac{\omega}{K}, \\
& \Phi^{-+-} \quad \rightarrow \quad \sin \left(-\phi_{1}+2 \phi_{2}\right)+\sin \phi_{1}=\frac{\omega}{K}, \\
& \left.\begin{array}{l}
\Phi^{--+} \\
\Phi^{+--} \\
\Phi^{---}
\end{array}\right\} \quad \rightarrow \quad \sin \phi_{1}=\frac{\omega}{2 K} .
\end{aligned}
$$

Since the set (42) corresponds to all possible solutions of equation (34a), every fixed point in the synchronized region can be represented by the functions $K^{-1}\left(\phi_{1}\right)$, obtained from (42) and (34b). The solutions, with their corresponding bifurcations point in each subspace represented by the pair $\left(K^{*}, \phi_{1}^{*}\right)$, are described in the table 1 and illustrated in figure 5 with $\omega=1$.

An unexpected feature that comes from the symmetry presented by the system concerns the characterization of the critical coupling: our analysis shows that $K_{s}$ is highly degenerate, since each subspace has two bifurcations responsible for the synchronization, a node-node bifurcation (with opposite stabilities) coming from the $\phi_{2}^{+}$solutions, always accompanied by a saddle-saddle or a saddle-(unstable)node coming from the $\phi_{2}^{-}$solutions. It is important to notice that not only at $K_{s}$ but at all values of the coupling constant where new solutions are born there exists the same composition of double bifurcations, as can be observed in figure 5. The reason why this feature comes as unexpected lies on the behavior of the 


\begin{tabular}{|c|c|}
\hline \multicolumn{2}{|r|}{ Subspace $\Phi^{+++}$} \\
\hline$\phi_{2}^{+}=\frac{2 \pi}{3} n_{1}-\phi_{1}$ & $\begin{array}{ll}n_{1}=0 & \rightarrow\left(K^{*}, \phi_{1}^{*}\right)=(\omega / 2, \pi / 2) \\
n_{1}=1 & \rightarrow\left(K^{*}, \phi_{1}^{*}\right)=(\omega, 5 \pi / 6) \\
n_{1}=-1 & \rightarrow\left(K^{*}, \phi_{1}^{*}\right)=(\omega, \pi / 6)\end{array}$ \\
\hline $\begin{aligned} \phi_{2}^{-}= & \pi\left(2 n_{2}-1\right) \\
& -3 \phi_{1}\end{aligned}$ & $n_{2}=0 \rightarrow\left\{\begin{array}{l}\left(K^{*}, \phi_{1}^{*}\right)=(\omega / 2, \pi / 2) \\
\left(K^{*}, \phi_{1}^{*}\right) \approx(1.84,3.56) \\
\left(K^{*}, \phi_{1}^{*}\right) \approx(1.84,-0.42)\end{array}\right.$ \\
\hline \multicolumn{2}{|r|}{ Subspaces $\Phi^{-++}$and $\Phi^{++-}$} \\
\hline$\phi_{2}^{+}=2 \pi n_{1}-\phi_{1}$ & $n_{1}=0 \rightarrow\left(K^{*}, \phi_{1}^{*}\right)=(\omega / 2, \pi / 2)$ \\
\hline$\phi_{2}^{-}=\frac{\pi\left(2 n_{2}+1\right)-\phi_{1}}{3}$ & $\begin{array}{l}n_{2}=0 \rightarrow\left(K^{*}, \phi_{1}^{*}\right) \approx(1.84,1.88) \\
n_{2}=1 \rightarrow\left(K^{*}, \phi_{1}^{*}\right) \approx(1.84,1.26) \\
n_{2}=2 \rightarrow\left(K^{*}, \phi_{1}^{*}\right)=(\omega / 2, \pi / 2)\end{array}$ \\
\hline \multicolumn{2}{|r|}{ Subspace $\Phi^{+-+}$} \\
\hline $\begin{array}{l}\phi_{2}^{+}=3 \phi_{1} \\
\phi_{2}^{-}=\pi-3 \phi_{1}\end{array}$ & $\begin{aligned}\left(K^{*}, \phi_{1}^{*}\right) & =(\omega / 2, \pi / 2) \\
\left(K^{*}, \phi_{1}^{*}\right) & \approx(1.84,-0.42) \\
\left(K^{*}, \phi_{1}^{*}\right) & \approx(1.84,3.56)\end{aligned}$ \\
\hline \multicolumn{2}{|r|}{ Subspace $\Phi^{-+-}$} \\
\hline$\phi_{2}^{+}=\frac{2 \pi n_{1}+\phi_{1}}{3}$ & $\begin{array}{ll}n_{1}=0 & \rightarrow\left(K^{*}, \phi_{1}^{*}\right) \approx(1.84,1.26) \\
n_{1}=1 & \rightarrow\left(K^{*}, \phi_{1}^{*}\right) \approx(1.84,1.88) \\
n_{1}=-1 & \rightarrow\left(K^{*}, \phi_{1}^{*}\right)=(\omega / 2, \pi / 2)\end{array}$ \\
\hline $\begin{aligned} \phi_{2}^{-}= & \pi\left(2 n_{2}-1\right) \\
& +\phi_{1}\end{aligned}$ & $n_{2}=0 \rightarrow\left(K^{*}, \phi_{1}^{*}\right)=(\omega / 2, \pi / 2)$ \\
\hline \multicolumn{2}{|c|}{ Subspaces $\Phi^{--+}, \Phi^{+--}$and $\Phi^{---}$} \\
\hline $\begin{array}{l}\phi_{2}^{+}=-\phi_{1} \\
\phi_{2}^{-}=-\pi+\phi_{1}\end{array}$ & $\left(K^{*}, \phi_{1}^{*}\right)=(\omega / 2, \pi / 2)$ \\
\hline
\end{tabular}

TABLE I: Solutions for each symmetric subspace and the respective bifurcation points. The solutions $\phi_{2}^{ \pm}\left(\phi_{1}\right)$ for each subspace (on the left) depend on integer numbers $n_{1,2}$. When the two solutions have the same bifurcation points $\left(K^{*}, \phi_{1}^{*}\right)$, the subspace is completely symmetric with respect to $\phi_{2}^{ \pm}$(except for the stability), and the number of bifurcations is doubled.

system when no symmetries are present, since the critical coupling was always obtained as a single bifurcation and the presence of double bifurcations was not addressed.

Although the solutions found on the subspaces correspond to all the fixed points of the system, the stability obtained from the eigenvalues of the bidimensional Jacobian matrix in equation (40) does not necessarily correspond to the stability of the solutions in the complete phase space. Thus, in order to know the complete stability of solutions it will be necessary to analyze the eigenvalues from the $(N-1) \times(N-1)$ Jacobian obtained from all the equations of motion instead of the bidimensional set. After carrying out the analysis on the fixed points born at $K_{s}$ we observed the existence of only one stable fixed point, originated from $\Phi^{+++}$: the stable solution from $\phi_{2}^{+}\left(n_{1}=0\right)$. All other fixed points born at $K_{s}$ were created from bifurcations saddle-saddle like in the complete phase space.

The result of the stability analysis computed in the region slightly above $K_{s}$ may be summarized as follows:

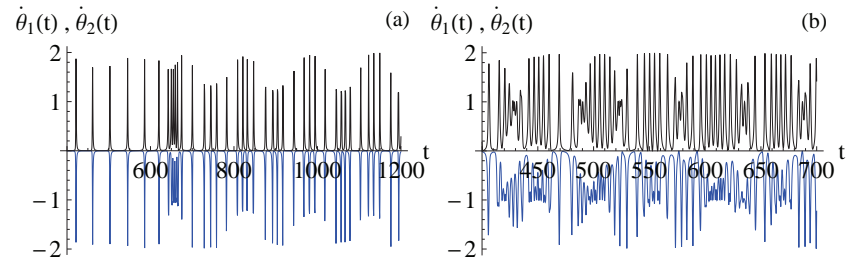

FIG. 6: Time evolution of the instantaneous frequencies $\dot{\theta}_{1}(t)$ and $\dot{\theta}_{2}(t)$ just below synchronization $(K=0.499)$ for a system with $N=6$ and specular symmetry with two natural frequencies and $\omega=1$. (a) For a set of initial conditions chaotic behavior of the time difference between slips is observed, with Lyapunov exponent approximately equal to 0.03. (b) Time evolution of the instantaneous frequencies for a set of oscillators which wander around phase space and only eventually find the ghost of the bifurcation.

from a total of 32 solutions created from 16 different bifurcations (remember that there exist 8 subspaces of symmetric solutions, each with two bifurcations), there is one stable fixed point originated from node-node bifurcations while the other 30 are saddles. If we consider a system with such a large number of saddles and one stable node, it would be only natural to expect an unusual behavior. With that in mind, we analyzed the time evolution of the instantaneous frequencies which is illustrated in figure 6. When synchronization is about to happen $(K=0.499)$ we expected to observe phase slips, with the period between slips proportional to $\left(K_{s}-K\right)^{\alpha 13,23}$. Surprisingly what we observed here is a strong dependence on the initial conditions: from some initial conditions the trajectories of the oscillators pass near the regions where the bifurcations appear, but as there are several possible bifurcations they get shifting from one to another (each producing a slip of a different strength) and the period between phase slips behaves chaotically (figure 6a). To characterize the behavior of this time difference between phase slips we calculate the largest Lyapunov exponent $\lambda^{24,25}$ which was found to be approximately 0.03 ; at the same time there are initial conditions that make the oscillators wander around phase space and only eventually find a neighborhood of a bifurcation, what does not necessarily happen simultaneously for all oscillators, resulting on erratic behavior and intermittent phase slips as seen in figure $6 \mathrm{~b}$. This unusual behavior is also observed for values of $K$ slightly above $K_{s}$ not always finding the stable fixed point in a reasonable time.

So far we have described the behavior of the system prior to and on a neighborhood immediately after the onset of synchronization. Now we will extend the discussion to the solutions in the synchronized region, that present some peculiarities coming from the high density of solutions. We shall focus our attention on the $\Phi^{++\ldots}$ subspace, which present most (if not all) of the system's features and has a direct link to the system's stability in the whole phase space which will be explained in the next section. 
For an arbitrary number $N$ of oscillators, the $\Phi^{++\ldots}$ representation of equation (34a) is given by

$$
\sin \left[\frac{N \phi_{1}+(N-2) \phi_{2}}{2}\right]+\sin \phi_{1}=\frac{\omega}{K} .
$$

Combining (43) with (34b), the variables $\phi_{1}$ and $\phi_{2}$ are related through the equation

$$
\sin \left[\frac{N \phi_{1}+(N-2) \phi_{2}}{2}\right]=-\sin \phi_{2}
$$

which admits two types of solutions:

$$
\begin{aligned}
& \phi_{2}^{+}=\frac{4 \pi m_{1}}{N}-\phi_{1}, \quad m_{1}=0, \pm 1, \pm 2, \ldots, \\
& \phi_{2}^{-}=\frac{2 \pi\left(2 m_{2}-1\right)-N \phi_{1}}{N-4}, \quad m_{2}=0,1,2, \ldots .
\end{aligned}
$$

For each type of solution (45) there exists a function $K_{ \pm}^{-1}\left(\phi_{1}\right)$ which describes the fixed points of the system,

$$
\begin{aligned}
& K_{+}^{-1}\left(\phi_{1}, m_{1}\right)= \\
& \quad \frac{1}{\omega}\left[\sin \phi_{1}\left(1+\cos \frac{4 \pi m_{1}}{N}\right)-\cos \phi_{1} \sin \frac{4 \pi m_{1}}{N}\right], \\
& K_{-}^{-1}\left(\phi_{1}, m_{2}\right)= \\
& \frac{1}{\omega}\left\{\sin \left[\frac{(N-2)\left(2 m_{2}-1\right) \pi-N \phi_{1}}{N-4}\right]+\sin \phi_{1}\right\},
\end{aligned}
$$

where the minima $\left.\partial_{\phi_{1}} K_{ \pm}\left(\phi_{1}\right)\right|_{\phi_{1}=\phi_{1}^{*}}=0$ (maxima of $\left.K_{ \pm}^{-1}\right)$ give the points $\phi_{m_{1}}^{*}$ and $\phi_{m_{2}}^{*}$ where the bifurcations occur,

$$
\begin{aligned}
\tan \phi_{m_{1}}^{*} & =-\frac{1+\cos \frac{4 \pi m_{1}}{N}}{\sin \frac{4 \pi m_{1}}{N}}, \\
\cos \phi_{m_{2}}^{*} & =\frac{N \cos \left[\frac{(N-2)\left(2 m_{2}-1\right) \pi-N \phi_{m_{2}}^{*}}{N-4}\right]}{N-4} .
\end{aligned}
$$

Figures $7 \mathrm{a}$ and $7 \mathrm{~b}$ show the solutions $K_{+}^{-1}\left(\phi_{1}, m_{1}\right)$ and $K_{-}^{-1}\left(\phi_{1}, m_{2}\right)$, respectively, with the stable branches in solid lines for $N=10$. The stability of the solutions is addressed via the eigenvalues of the 2-dimensional Jacobian matrix, and we see that the systems exhibits: saddle(stable)node bifurcations for $m_{1}=1$ and 2, (stable)node(unstable)node for $m_{1}=0$, saddle-(unstable)node for $m_{1}=-1$ and -2 for the case $K_{+}^{-1}\left(\phi_{1}, m_{1}\right)$. For the figure $7 \mathrm{~b}$ we obtain: saddle-(stable)node bifurcations for $m_{1}=0$ and 2 on the left, saddle-(unstable)node for $m_{2}=0$ and 2 on the right, and a saddle-saddle $m_{2}=1$.

The first feature that draws attention in figure 7 is the presence of changes in the stability of a given branch when the coupling varies (see $m_{1}=-1$ and -2 and the left branches for $m_{2}=0$ and 2). From the real part of the eigenvalues (figure 8) we could see that $\lambda_{-}\left(\phi_{1}\right)$ is responsible for the inversions: at the bifurcations of $m_{1}=$ -1 and -2 the fixed point is born as a saddle $\left(\operatorname{Re}\left[\lambda_{+}\right]<0\right.$
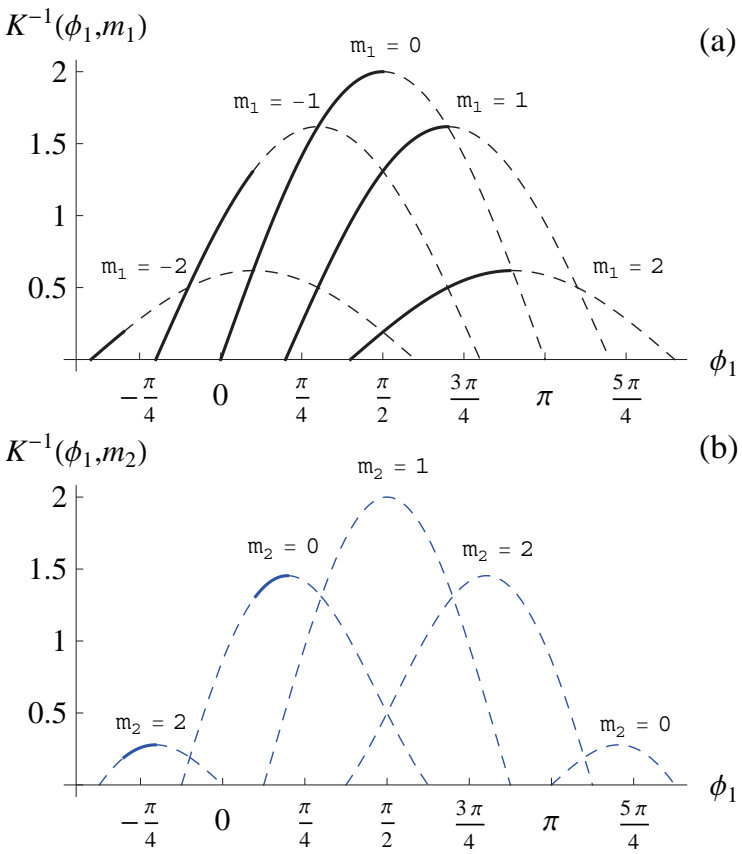

FIG. 7: (Color online) The plots of $K_{ \pm}^{-1}\left(\phi_{1}, m_{1,2}\right)$ representing the fixed points of the system synchronized region with two natural frequencies and $N=10$. Solid lines (dashed) represent stable fixed points (unstable). (a) Solutions of $K_{+}^{-1}\left(\phi_{1}, m_{1}\right)$ with bifurcations type node-node $m_{1}=0$; saddle-(stable) node bifurcation for $m_{1}=1$ and $m_{1}=2$, and saddle- (unstable) node for $m_{1}=-1$ and $m_{1}=-2$. (b) solutions $K_{-}^{-1}\left(\phi_{1}, m_{2}\right)$, with saddle-saddle bifurcations $m_{2}=1$, saddle-(stable)node bifurcation in the left of $m_{2}=0$ and $m_{2}=2$; saddle-(unstable)node bifurcation in the right of $m_{2}=0$ and $m_{2}=2$.

and $\left.\operatorname{Re}\left[\lambda_{-}\right]>0\right)$, but then it changes into a stable node at the point where the real part of $\lambda_{-}$becomes negative (figures $8 \mathrm{a}$ and $8 \mathrm{~b}$ ). For the bifurcations on the left of $m_{2}=0$ and 2 , shown in figure $7 \mathrm{~b}$, the real part of $\lambda_{-}$is positive and becomes negative after a given value of $\phi_{1}$, where the saddles become stable nodes (figures 8 r and 8d).

The exact value of the points where the branches change stability can be identified if we analyze all those solutions simultaneously as in figure 9. the branch born from the bifurcation on the left of $m_{2}=0$ loses stability when it touches the branch born at $m_{1}=-1$ for $K \approx 0.764$ with $\phi_{1}=\pi / 10$, which becomes stable when the coupling increases (figure 9a); the same process occurs with the branch coming from the bifurcation on the left of $m_{2}=2$, which exchanges stability with the $m_{1}=-2$ solution at $K \approx 5.236$ and $\phi_{1}=-3 \pi / 10$ (figure 9b). The conclusion from this analysis is that a transcritical bifurcation is indeed responsible for the changes in the stability of the fixed points.

Changes in the stability properties of the fixed points occur in the stable as well as the unstable branches as can be seen in the figures $8 \mathrm{r}$ and $8 \mathrm{~d}$ : at the saddle(unstable)node bifurcations on the right of $m_{2}=0$ and 


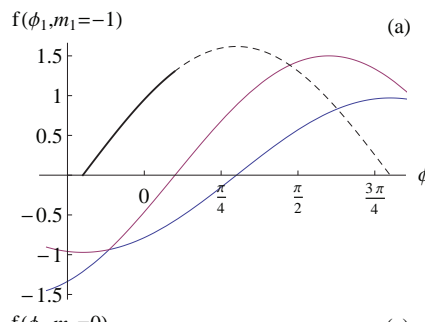

(a) $\left(\phi_{1}, m_{1}=-2\right)$

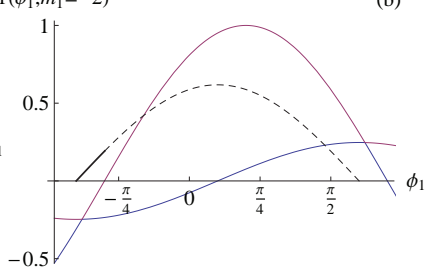

$\mathrm{f}\left(\phi_{1}, m_{2}=0\right)$

(c) $\mathrm{f}\left(\phi_{1}, m_{2}=2\right)$
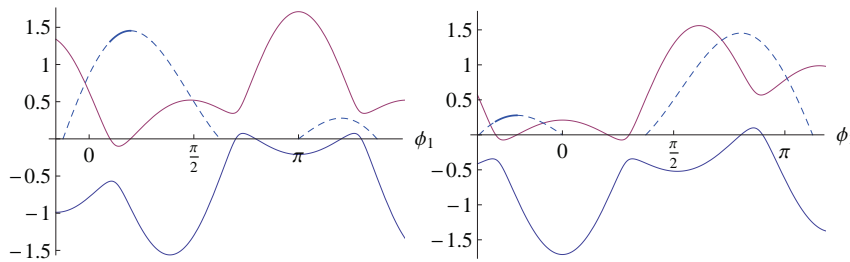

FIG. 8: (Color online) Branches of solutions for the system with $N=10$ and two natural frequencies, that have changed the stability by varying the strength of the coupling K. Stable solutions (unstable) are represented by solid lines (dashed). Upper continuous curves (purple) represents the real part of $\lambda_{-}\left(\phi_{1}\right)$, and down curves (blue) represent the real part of $\lambda_{+}\left(\phi_{1}\right)$. Figures (a) and (b): bifurcations labeled by $m_{1}=-1$ and $m_{1}=-2$, respectively, showing that the solutions are born on the right are saddles and become stable node when we the real part of $\lambda_{-}$becomes negative. Figures (c) and (d): bifurcations labeled by $m_{2}=0$ and $m_{2}=2$, respectively, showing that the solutions are born on the left are stable nodes and become saddles when the real part of $\lambda_{-}$becomes positive.
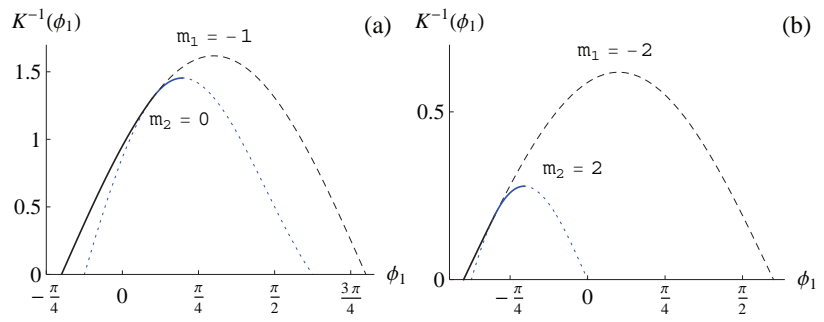

FIG. 9: (Color online). Region where solutions exchange stability via a transcritical bifurcation for the cases $K_{ \pm}^{-1}\left(\phi_{1}\right)$. (a) Stable node denoted by $m_{2}=0$ loses stability to the saddle denoted by $m_{1}=-1$ at the intersection of the solutions. (b) Stable node $m_{2}=2$ loses stability to the saddle of $m_{1}=-2$ in intersection of the solutions. Solid(dashed) lines correspond to stable(unstable) solutions.(Both figures are done for a system with two natural frequencies and $N=10$ )

$m_{2}=2$, the unstable nodes become saddles when the real part of $\lambda_{+}$becomes negative. Since the solutions $K_{ \pm}^{-1}\left(\phi_{1}\right)$ are symmetric with respect to the $\phi_{1}=\pi / 2$ axis, the mechanism which alters the stability of those solutions is the same process as described before, with the exchanging points given by $\phi_{1}=9 \pi / 10$ and $\phi_{1}=13 \pi / 10$.

The stability exchange via a transcritical bifurcation may also take place on the complete phase space of the system, although the exchange is not necessarily made between solutions of the same subspaces. Therefore to obtain all the stable solutions of the system, independent of the size of their basins of attraction, it is necessary to obtain all the points in space where two (or more) solutions collide and to analyze the stability of those fixed points before and after the collision (while at the same time the stability of all the other solutions should be considered). Since the number of solutions is exceedingly large to handle, even for small systems, this type of symmetry may not be the most enlightening choice to obtain a description of the synchronized region in a simple (analytical) fashion. So in the next section we will restrict our symmetry assumptions to more general cases, looking for the analyticity limits of the model.

\section{SPECULAR SYMMETRY}

Let us consider an even number of oscillators with a configuration of frequencies that satisfy the following symmetry:

$$
\omega_{N / 2+n}=-\omega_{n}, \quad n=1, . ., N / 2,
$$

and also consider that all natural frequencies in the interval $\left[\omega_{1}, \omega_{N / 2}\right]$ are positive. If we write the variables $\phi_{n}$ in terms of $\phi_{N / 2}$,

$$
\sin \phi_{n}=\sin \phi_{N / 2}-\frac{1}{K} \sum_{j=n+1}^{n^{*}} \omega_{j}
$$

for $n=1, \ldots, N / 2-1$ and

$$
\sin \phi_{n}=\sin \phi_{N / 2}+\frac{1}{K} \sum_{j=N / 2+1}^{n} \omega_{j},
$$

for $n=N / 2+1, \ldots, N-1$, the synchronized region is determined by the solutions of equation (5) in the form:

$$
\sin \phi_{N / 2}-\sin \phi_{N}\left(\phi_{N / 2}\right)=\frac{\sum_{n=1}^{N / 2} \omega_{n}}{K} .
$$

From the analysis of the symmetry properties it is possible to conclude that there is a solution of this equation with $\phi_{n+N / 2}=-\phi_{n}$ as long as $\sin \phi_{N}=-\sin \phi_{N / 2}$. Since the last identity maximizes the left hand side of equation (50), the critical synchronization coupling is given by

$$
K_{s}=\frac{\sum_{j=1}^{N / 2} \omega_{j}}{2} .
$$

The determination of the critical coupling with this symmetry is reduced to adding the first $N / 2$ values of the natural frequencies, therefore any prescription that we choose to determine the natural frequencies $\omega_{n}(N)(n=$ $1, \ldots, N / 2)$ will allow us to obtain the dependence of $K_{s}$ on the size of the system $N$. For instance, if we consider a general case where the frequencies in the interval 

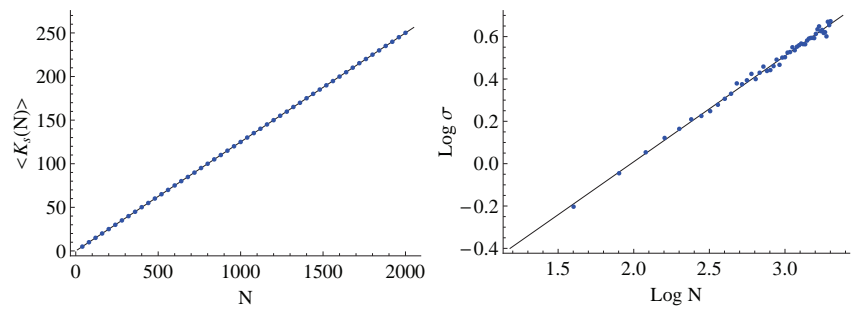

FIG. 10: Left: behavior of $\left\langle K_{s}(N)\right\rangle$ for natural frequencies generated by a uniform distribution with $a=0$ and $b=1$ and systems with specular symmetry. Right: standard deviation $\sigma\left[K_{s}(N)\right]$ from the numeric outcome in logarithmic scale. The dots correspond to the result obtained through the simulation, with average obtained from 1000 instances. The black line correspond to the curve calculated via (52).

$\left[\omega_{1}, \omega_{N / 2}\right]$ are obtained from a uniform distribution defined on the interval $[a, b]$, with $a>0$ and $b>a$, it is easy to compute the mean of $K_{s}(N)$ and the standard deviation from equation (51):

$$
\left\langle K_{s}(N)\right\rangle=\frac{a+b}{8} N, \quad \sigma\left[K_{s}(N)\right]=\frac{b-a}{4 \sqrt{6}} \sqrt{N} .
$$

These results are corroborated by the simulation as illustrated in figure 10. A statistical study of the solutions for the local Kuramoto model with quenched disorder has not yet been addressed in the literature. Here we show the average critical coupled strength $K_{s}$ when the random field have specular symmetry. The analytical solutions for this systems depends on the possibility to identify the maximum sequential sum of the frequencies inside the ring 11 . Analytical solutions for $K_{s}$ can be obtained for all symmetries that allow this identification.

Although the imposition of the symmetry defined in (48) on the set of natural frequencies allowed us to determine $K_{s}$ analytically, there is still the problem of finding all the solutions in the synchronized region. The selection of the phase difference $\phi_{N / 2}$ as the independent variable warrants that no sum in the equations (49) will be larger than the sum on the right hand side of (50). From this we infer that there exists a region of values of $K$ such that the set (49) has a solution (the right hand side of each equation always belongs to the interval $[-1,1]$ ) while $\phi_{N / 2}$ does not satisfy (50). This means that all the solutions in the synchronized region exist after a value $K=K_{s}$ which guarantees the existence of a solution for equation (50), while in the synchronized region $\left(K \geq K_{s}\right)$ the solutions of the form $\phi_{N / 2}(K)$ correspond to the fixed points of the system.

To get all the solution in terms of $\phi_{n}\left(\phi_{N / 2}\right)$ we have to consider the equations (49) that define relations between

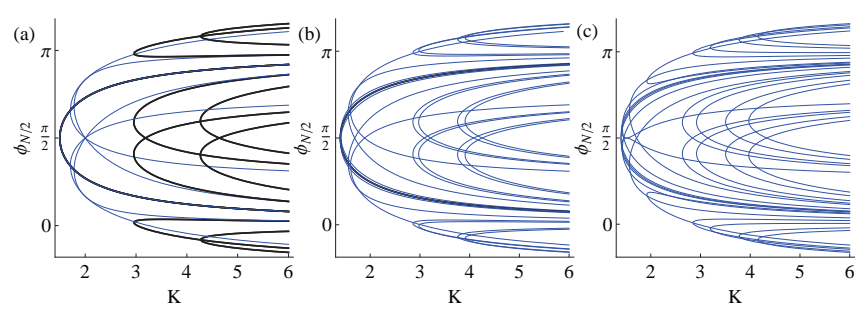

FIG. 11: (Color online) Numerical solution of equation (53) in the space $\phi_{N / 2} \times K$ representing the fixed points of the system with $N=6$ oscillators. (a) Setting $\omega_{n}=\omega$ for $\left(\omega_{1}, \omega_{2}, \omega_{3}\right)$ with high degeneracy and specular symmetry. (b) The presence symmetry given by (48) separates the solutions such that only the bifurcations located in $K_{s}$ are degenerated. The natural frequencies are $\omega_{1}=1, \omega_{2}=0.7$ and $\omega_{3}=1.1$.(c) The continuous symmetry breaking process separates the spectrum of solutions completely, destroying the degeneracy. The natural frequencies are $\omega_{1}=1, \omega_{2}=0.7, \omega_{3}=1.1, \omega_{4}=-0.8$, $\omega_{5}=-0.75$ and $\omega_{6}=-1.25$.

the phases, thus all possible combinations:

$$
\begin{aligned}
& \phi_{n}^{+}=\arcsin \left(\sin \phi_{N / 2}-\frac{1}{K} \sum_{j=n+1}^{n^{*}} \omega_{j}\right) \\
& \phi_{n}^{-}=\pi-\arcsin \left(\sin \phi_{N / 2}-\frac{1}{K} \sum_{j=n+1}^{n^{*}} \omega_{j}\right)
\end{aligned}
$$

for $n=1, \ldots, N / 2-1$, and

$$
\begin{aligned}
& \phi_{n}^{+}=\arcsin \left(\sin \phi_{N / 2}+\frac{1}{K} \sum_{j=N / 2+1}^{n} \omega_{j}\right) \\
& \phi_{n}^{-}=\pi-\arcsin \left(\sin \phi_{N / 2}+\frac{1}{K} \sum_{j=N / 2+1}^{n} \omega_{j}\right)
\end{aligned}
$$

for $n=N / 2+1, \ldots, N-1$, should be considered a priori ${ }^{31}$. In order to correctly describe the systems we shall start with simple frequency configurations.

The simplest configuration of frequencies satisfying the symmetry (48) is $\omega_{n}=\omega$ for $n=1, \ldots, N / 2$, with critical coupling given by

$$
K_{s}(N)=\frac{\omega N}{4}
$$

If we consider a small system $N=6$, all $2^{N-2}=16$ combinations of the kind of solutions (53) may be considered to solve (50) numerically, as shown in figure 117. Even though it is possible to determine all solutions for $\phi_{N / 2}=\phi_{3}$ above $K_{s}$, the high symmetry level presents the same problem of degeneracies in the $\phi_{N / 2} \times K$ space found in the previous section: there are several values of phase locking $\phi_{1}, \phi_{2}, \phi_{4}$ and $\phi_{5}$ which lead to the same result for $\phi_{3}$. In larger systems the process of assigning an observed fixed point to a phase locked solution is practically impossible. However there is no need for special 
prescription for the natural frequencies within $\left[\omega_{1}, \omega_{N / 2}\right]$ if we are looking for solutions that satisfy (48). Even if we consider a case with complete broken symmetry, equation (50) will still be valid as long as the sum in the right hand side continues to be larger than all other sums in (49).

Since small perturbations in the natural frequencies have little effect in the synchronized region, it is possible to start from the configuration that produces equation (54) and continuously vary the frequencies while following the solutions in the $\phi_{N / 2} \times K$ space. If we do this while preserving the specular symmetry (48), we observe that all solutions born after $K_{s}$ are separated (as can be seen in figure 11b for $\omega_{1}=1, \omega_{2}=0.7$ and $\omega_{3}=1.1$ ). If we continue this process until the configurations satisfy only equation (50) (without symmetries), the degeneracy of the solutions is destroyed (the figure 11: shows a case with $\omega_{1}=1.0, \omega_{2}=0.7, \omega_{3}=1.1, \omega_{4}=-0.8$, $\omega_{5}=-0.75$ and $\omega_{6}=-1.25$, where several solutions exist above $K_{s}$ but without any overlaps).

The continuous symmetry breaking of equation (48) illustrates a property present in any configuration of natural frequencies: the presence of degeneracies in the space $\phi_{n^{*}} \times K$ is a consequence of the symmetries in the set $\{\omega\}_{N}$. The importance of this property becomes evident when one consider a system with asymmetric frequency configuration: in general the main interest does not lie on the description of the full set of solutions above $K_{s}$, but on the determination of the stable fixed points. While for a chain of oscillators the stable fixed point is characterized by $\cos \phi_{n}>0$ (for all $n$ ), the loop structure of the ring allows the existence of stable fixed points with $\cos \phi_{n}<0$ for some $n$ (see Lee et al. ${ }^{26}$ ), and our recent work ${ }^{11}$ showed that these phase differences may either be $\phi_{n^{*}}$ or $\phi_{N}$. Hence it is only necessary to analyze the solutions of (50) with relations $\phi_{n}\left(\phi_{N / 2}\right)$ defined by (53a) and (53c), i.e., $\phi_{n} \in[-\pi, \pi]$ for $n \neq n^{*}$, while all other combinations of (53) always lead to unstable solutions. Now it becomes clear the choice for the $\Phi^{++\ldots}$ in the previous chapter: all stable fixed points for the dynamics on the whole phase space come from the solutions of this specific subspace.

A general characterization of the synchronized region in the absence of symmetries (or with a small amount) was already done in our previous work ${ }^{11}$, therefore it is not necessary to do it again. Nevertheless we can use the symmetry properties of the natural frequencies in order to study the behavior of the critical synchronization coupling $K_{s}$ as a function of the number of oscillators, as discussed in the next section.

\section{ASYMPTOTIC BEHAVIOR OF THE CRITICAL COUPLING}

Possibly the simplest non-degenerate case analyzable through the solutions of (50) consists in the configuration where the frequencies are evenly spaced in the interval

$$
\begin{aligned}
& {[-\gamma, \gamma]:} \\
& \quad \omega_{n}=\frac{\gamma}{N-1}(-2 n+N+1), \quad n=1, \ldots, N .
\end{aligned}
$$

The frequencies of the oscillators are mirror images with respect to the axis between $\phi_{N}$ and $\phi_{N / 2}$, i. e., $\omega_{N+1-n}=$ $-\omega_{n}$ for $n=1, \ldots, N / 2$, which gives

$$
\sin \phi_{N / 2+n}=\sin \phi_{n}, \quad n=1, \ldots, N / 2-1 .
$$

Assuming $\phi_{N / 2+n}=\phi_{n}$ the phase differences $\phi_{n}$ in (53) are given by:

$$
\phi_{n}\left(\phi_{N / 2}, K\right)=\arcsin \left[\sin \phi_{N / 2}-\frac{\gamma(N-2 n)^{2}}{4(N-1) K}\right],
$$

for $n=1, \ldots, N / 2-1$, such that the solutions in the synchronized region are obtained from

$$
\sin \left(\phi_{N / 2}+2 \sum_{n=1}^{\frac{N}{2}-1} \phi_{n}\right)+\sin \phi_{N / 2}=\frac{\gamma N^{2}}{4(N-1) K} .
$$

The bifurcation responsible for the full synchronization is always the first solution of (58) and it can appear near any of the two boundaries of the solvability region, i.e., it is characterized either by $\sin \phi_{N / 2} \approx 1$ or $\sin \phi_{N} \approx$ -1 . The number of bifurcations giving birth to stable solutions is proportional to the number of oscillators, and for increasing $N$ we observed that they tend to pile up near the minimum value of $K$ under which equation (58) may present a solution. In other words, for large $N$ it is possible to approximate $\sin \phi_{N / 2}-\sin \phi_{N} \approx 2$ to obtain an asymptotic behavior for the critical coupling:

$$
K_{s}^{\text {asymptotic }}(N)=\frac{\gamma N^{2}}{8(N-1)}
$$

Figure $12 \mathrm{a}$ shows the results obtained from the simulation in contrast to the analytical expression in (59), while figure 12b exhibits the actual value of the sum of the two sines, thus corroborating the used approximation.

That asymptotic behavior of $K_{s} \sim N$, also observed in the cases treated previously, is not just a consequence of the evenly spaced frequencies but rather from the fact that the interval is finite, thus the differences $\omega_{n}-\omega_{n+1}$ are proportional to $1 / N$. If we consider the configuration

$$
\omega_{n}=\frac{\epsilon}{2}(-2 n+N+1), \quad n=1, \ldots, N,
$$

where $\omega_{n}-\omega_{n+1}=\epsilon$, independent of $N$, the equations (57) and (58) are written as

$$
\phi_{n}\left(\phi_{N / 2}, K\right)=\arcsin \left[\sin \phi_{N / 2}-\frac{\epsilon(N-2 n)^{2}}{8 K}\right],
$$

for $n=1, \ldots, N / 2-1$, and

$$
\sin \left(\phi_{N / 2}+2 \sum_{n=1}^{\frac{N}{2}-1} \phi_{n}\right)+\sin \phi_{N / 2}=\frac{\epsilon N^{2}}{8 K} .
$$



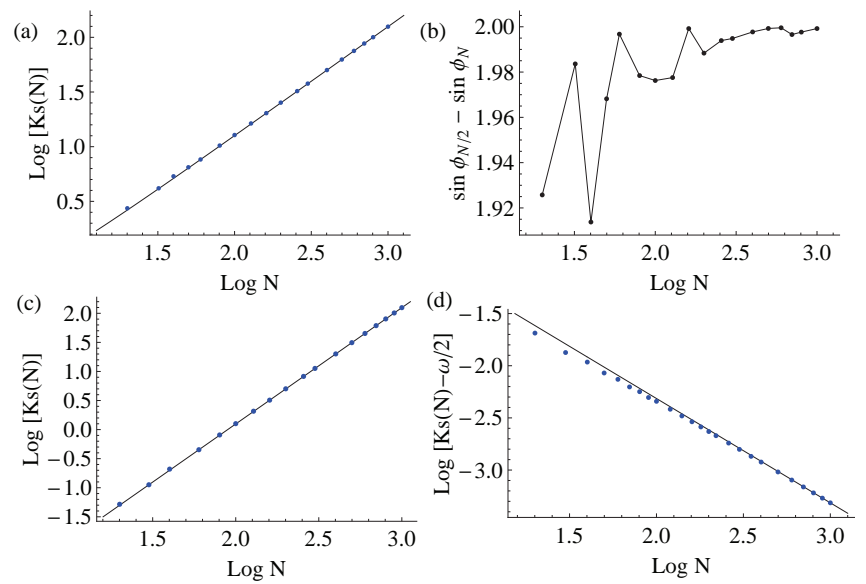

FIG. 12: Behavior of $K_{s}(N)$ obtained from different configurations of natural frequencies, represented by dots, and the continuous line are the theoretical asymptotic curves: a) $\omega_{n}$ is defined in (55) with $\gamma=1$ (logarithmic scale) and the line is given by equation (59). b) Behavior of $\sin \phi_{N / 2}-\sin \phi_{N} \approx 2$. c) $\omega_{n}$ is defined in (60) with $\epsilon=0.002$ (logarithmic scale) and the line is given by the equation (63). d) $\omega_{n}$ is defined in (65) with $\gamma=1$, and the coupling is inversely proportional to the system size. The curve corresponds to the asymptotic fitting (68) with $A=0,485$.

Since this system presents the same characteristics as the latter, the asymptotic limit of $K_{s}(N)$ can also be obtained with $\sin \phi_{N / 2}-\sin \phi_{N} \approx 2$, so

$$
K_{s}^{\text {asymptotic }}(N)=\frac{\epsilon N^{2}}{16},
$$

as shown in figure 12 .

For all the analyzed cases, the critical value of the coupling constant at synchronization grows with $N$, which is common in systems with nearest neighbor interactions between oscillators, and this includes the chain of oscillators $^{23}$. In both cases given by (48) as well as the mirror symmetry $\left(\omega_{N+1-n}=-\omega_{n}\right)$ present in the evenly spaced configurations of frequencies (55) and (60), any prescription for the distribution of frequencies that has a well defined behavior of the sum of the frequencies with $N$, that is,

$$
\sum_{n=1}^{N / 2} \omega_{n}=f(N)
$$

will have (in general) a behavior of the critical coupling of the form $K_{s}(N) \sim \frac{1}{2} f(N)$, being exact for (48) and asymptotic for the other cases. As in general the sum in (64) produces a function which increases with $N$, these configurations will always have $K_{s} \rightarrow \infty$ when $N \rightarrow \infty$. But, there exists at least one configuration for which this property is not valid: a system with mirror symmetry $\omega_{N+1-n}=-\omega_{n}$ and natural frequencies given by

$$
\omega_{n}=\frac{\gamma(-1)^{n+1}}{N-1}(-2 n+N+1), \quad n=1, \ldots, N / 2 .
$$

Due to the term $(-1)^{n+1}$ the largest sequential sum $\sum_{n} \omega_{n}$ that can be obtained corresponds to the largest element of the set $\{\omega\}_{N}\left(\omega_{1}=\gamma\right)$, such that the equations that describe this system in the synchronized region are:

$\phi_{n}\left(\phi_{1}\right)=\arcsin \left\{\sin \phi_{1}-\frac{\gamma\left[N-2+(-1)^{n}(N-2 n)\right]}{2(N-1) K}\right\}$,

for $n=2, \ldots, N / 2$, and

$$
\sin \left[2 \phi_{1}+2 \sum_{n=2}^{N / 2-1} \phi_{n}\left(\phi_{1}\right)+\phi_{N / 2}\left(\phi_{1}\right)\right]+\sin \phi_{1}=\frac{\gamma}{K} .
$$

When the number of oscillators increases the effect in the solvability region is the same as that in the cases of evenly spaced frequencies, therefore in the limit $N \rightarrow$ $\infty$ we must have $\sin \phi_{1}-\sin \phi_{N} \sim 2$. But as the right hand side of equation (67) does not depend on $N$, the critical coupling $K_{s}$ should decrease with $N$ (since for small systems $K_{s}>\gamma / 2$ ). Then, if we take the limit of a very large number of oscillators, we should obtain

$$
K_{s}^{\text {asymptotic }}(N)=\gamma / 2+\frac{A}{N}+\mathrm{O}\left(N^{-2}\right),
$$

as can be seen in figure $12 \mathrm{~d}$, where $A$ is just a constant. The importance to obtain a configuration of frequencies which produces a behavior for the critical coupling $K_{s} \sim 1 / N$ in the LCKM resides in the comparison with systems that possess larger network connectivity. The critical coupling in the Kuramoto model (globally connected) decreases with $N$ for natural frequencies defined in a finite interval ${ }^{28}$, while locally connected model seems to diverge in the limit $N \rightarrow \infty$. At first sight, one would expect a transition in the asymptotic limit for some critical network connectivity, but our result indicates that, under a proper organization of the natural frequencies in the LCKM, the two regimes may be connected in a continuous way, just as the type of interaction considered in ${ }^{29}$. We believe that this subject deserves further investigation.

\section{CONCLUSIONS}

In the present work we gave a comprehensive description of the locally coupled Kuramoto model under the presence of symmetries and explored to a large extent the analyticity limits of the system. In the following we summarize the salient features.

Starting with the analysis of small systems, we gave a full description of the synchronized region, along with the determination of the critical synchronization coupling for some cases, and we showed how the fixed points may be obtained from a general configuration of natural frequencies, independent of the system size.

To keep our attention on the analytical regime of the model, we considered a highly symmetric configuration, 
consisting of two natural frequencies: $\omega$ for odd numbered oscillators and $-\omega$ for even numbered ones. Despite of its apparent simplicity, many unusual and (or) unexpected results were observed.

We found that the stability of the fixed points depend on the number of dimensions of the manifold where the dynamics is set to happen: while for 2-dimensional systems (obtained from symmetric initial conditions) a large number of stable fixed points populate the phase space, the complete system is characterized by just a small amount of stable fixed points.

Several times we chose initial condition with a given symmetry. This was done to control the final state. As an example, consider the case of a system with two natural frequencies. The conditions given by equation (32) lead to equation (33). We note that the phase differences depend on two independent phases $\phi_{1}$ and $\phi_{2}$. All the solutions are constructed from all possible combinations of $\phi_{n}^{+}$and $\phi_{n}^{+}$in equation (37). So given some specific initial condition prescribed by equation (37), the solutions is already determined by equation (39). If we start with a set of initial conditions without the symmetry of the system, we cannot anticipate into which solution of equation (39) the dynamics will evolve. Imposing the symmetry on the phases from the beginning, we select the attractor in the corresponding subspace.

At the onset of synchronization it is well known that one should expect periodic phase slips, however in the two frequencies domain the system exhibits a strong dependence on the initial conditions: a regime of phase slips with irregular period (apparently chaotic) coexists with complete erratic bursting behavior characterized by a long time absence of phase slips as well as intermittent appearances. This local character of the system also extends to the synchronized region, where we could observe that the stable fixed points created slightly above $K_{s}$ behave only as local attractors in the phase space. The coexistence of stable fixed points may lead to the appearances of attractor crowding, as described by Wiesenfeld and Hadley ${ }^{30}$, and we hope further investigation will clarify the issue.

We also observed stability exchange between synchronized solutions. This feature happens when two fixed point collide in the phase space. The values of $\phi$ for these exchanges to occur and the nature of each fixed point were determined through the analysis of the Jacobian's eigenvalues in the reduced system, and the same feature is expected to happen in the complete phase space.

The presence of a type of specular symmetry enabled us to obtain some analytical expressions for the critical synchronization coupling, but one important result came from the analysis of a continuous symmetry breaking of the system: the degeneracies in the $\phi_{n^{*}} \times K$ space are lifted in the absence of symmetries on the natural frequencies. As a consequence we could show how just a small amount of the solutions will be stable, and the search for stable fixed points may be reduced to the analysis of just the solutions composed by phase differences belonging to the interval $[-\pi, \pi]$.

By further exploring the symmetry properties we could manage to obtain some asymptotic results concerning the behavior of the critical synchronization coupling as a function of the system size $N$. While for general configuration the LCKM tends to be proportional to the number of oscillators, we found a counterexample where $K_{s}$ actually decreases with $N$.

Many of the phenomena and results discussed here were not observed in a completely random natural frequencies distribution. The study of all theses symmetric cases have brought insights about the complexity of the LCKM and helped us advance in the understanding and the development of analytical methods to analyze this kind of system.

\section{Acknowledgments}

P.F.C.T. acknowledges support by FAPESP and CAPES (Brazil).
${ }^{1}$ H. Sakaguchi, S. Shinomoto and Y. Kuramoto, Prog. Theot. Phys. 77 (1987) 1005.

2 K. Wiesenfeld, P. Colet and S. H. Strogatz, Phys. Rev. E 57 (1998) 1563

3 B. C. Daniels, S. T. M. Dissanayake, and B. R. Trees, Phys. Rev. E 67 (2003) 026216.

4 T. Miyano and T. Tsutsui, Phys. Rev. Lett. 98 (2007) 024102 .

${ }^{5}$ D. Cumin, C.P. Unsworth, Physica D 226 (2007) 181.

6 T.D. Frank, A. Daffertshofer, C.E. Peper, P.J. Beeka and H. Haken, Physica D 144 (2000) 62.desigualdad social

7 R.C. Moioli, P.A. Vargas and P. Husbands,Evolutionary Computation (CEC), 2010 IEEE Congress on, (2010) 2483.

8 J.J. Collins and I.N. Stewart, J. Nonlinear Sci. 3 (1993) 349.

9 J.A. Rogge and D. Aeyels, J. Phys.: Math. Gen. A 37
(2004) 11135

10 Z. Liu, Y.-C. Lai and F. C. Hoppensteadt, Phys. Rev. E 63 (2001) 055201(R).

11 P. F. C. Tilles, F. F. Ferreira and H. A. Cerdeira, Phys. Rev. E 83 (2011) 066206.

12 J. Bridges, R. Rand and S. M. Sah, Comm. Nonlin. Sci. Num. Sim. 14 (2009) 3901.

${ }^{13}$ H. F. El-Nashar, P. Muruganandam, F. F. Ferreira and H. A. Cerdeira, Chaos 19 (2009) 013103.

14 Zhigang Zheng, Gang $\mathrm{Hu}$, and Bambi Hu, Phys. Rev. Lett. 81 (1998) 5318.

15 H. F. El-Nashar and H. A. Cerdeira, Chaos 19 (2009) 033127.

16 P. Muruganandam, F. F. Ferreira, H. F. El-Nashar and H. A. Cerdeira, Pramana 70 (2008) 1143.

17 J. Rogge and D. Aeyels, J. Phys. A 37 (2004) 11135. 
18 J. Ochab , P.F. Gora, Acta Physica Polonica B Proceedings Supplement 3 (2010) 453.

19 M. Golubitsky, I. Stewart, and D. G. Schaeffer. Singularities and Groups in Bifurcation Theory", Volume II. Springer-Verlag, New York (1988).

20 Y. Maistrenko, O. Popovych, O. Burylko and P. A. Tass, Phys. Rev. Lett. 93 (2004) 084102.

${ }^{21}$ Y.L. Maistrenko, O. V. Popovych and P. A. Tass, Int. J. Bif. Chaos 15 (2005) 3457.

${ }^{22}$ P. Ashwin, O. Burylko and Y. Maistrenko, Physica D 237 (2008) 454.

23 S.H. Strogatz and R.E.Mirollo, Physica D 31 (1988) 143.

24 M.T. Rosenstein, J. J. Collins and C. J. De Luca, Physica D 65 (1993) 117.

25 M. B. Kennel, R. Brown, and H. D. I. Abarbanel, Phys. Rev. A 45 (1992) 3403.
26 T. E. Lee, H. Tam, G. Refael, J. L. Rogers and M. C. Cross, Phys. Rev. E 82 (2010) 036202.

27 J. Kennedy and J. A. Yorke Physica D 51 (1991) 213.

28 D. Pazó, Phys. Rev. E 72 (2005) 046211.

29 J. L. Rogers and L. T. Willie, Phys. Rev. E 54 (1996) R2193.

30 K. Wiesenfeld and P. Hadley, Phys. Rev. Lett. 62 (1989) 1335.

31 A certain amount of care should be considered when dealing with equation (53): the solutions $\phi_{n}^{-}$were written as $\pi-\arcsin (*)$ just for a easier presentation. It is necessary to know the quadrant where the argument is located to obtain the correct solution in the opposite quadrant that also satisfy (49). 